Editor's Note: These short reviews of a recent paper in the Journal, written exclusively by graduate students or postdoctoral fellows, are intended to mimic the journal clubs that exist in your own departments or institutions. For more information on the format and purpose of the Journal Club, please see http://www.jneurosci.org/misc/ifa_features.shtml.

\title{
The Neural Construction of a Tinkertoy
}

\author{
Roel M. Willems \\ F. C. Donders Centre for Cognitive Neuroimaging, Radboud University Nijmegen, 6500 HB Nijmegen, The Netherlands \\ Review of Frey and Gerry (http://www.jneurosci.org/cgi/content/full/26/51/13194)
}

We often learn by watching other people. Consider for example how children learn to lace their shoes. An adult will, granted enough patience, repeatedly demonstrate how to handle the shoe laces to get to the wanted end result. Although the demonstration will be accompanied by speech ("now bring this lace to the other side"), the observation of the sequence of motor acts is instructive for children to learn how to do it themselves. In adulthood such observational learning is also prevalent. Just think of someone showing you how to serve a tennis ball, how to dance, or how to open a bottle of wine. What these situations have in common is that the learner tries to learn a skill by observing how someone else performs this skill. Put differently, the learner tries to internalize the act that she observes so that she will be able to do it herself. In their recent study in The Journal of Neuroscience, Frey and Gerry (2006) investigated the brain mechanism underlying observational learning using functional magnetic resonance imaging. They were particularly interested in the role of the "action recognition system" in learning a sequence of acts by observation. In other words, would the observer's action recognition system be activated differently if an observed sequence had to be acted out later or not? Moreover, the authors looked into how

Received Jan. 2, 2007; revised Jan. 3, 2007; accepted Jan. 3, 2007.

Correspondence should be addressed to Roel M. Willems, F. C. Donders Centre for Cognitive Neuroimaging, Radboud University Nijmegen, P.O. Box 9101, 6500 HB Nijmegen, The Netherlands. E-mail: roel.willems@fcdonders.ru.nl.

DOI:10.1523/JNEUROSCI.0005-07.2007

Copyright $\odot$ 2007 Society for Neuroscience $\quad$ 0270-6474/07/271509-02\$15.00/0 focusing on the sequence of observed events affected parts of the neural network involved in observational learning.

Healthy participants watched a video clip of a person constructing a Tinkertoy [Frey and Gerry (2006), their Fig. 1 (http://www.jneurosci.org/cgi/content/ full/26/51/13194/F1)]. In the LEARN condition, participants were instructed to carefully watch the video and were asked to assemble the toy themselves after the experiment in exactly the same sequence of events as in the video. In the CONTROL condition, a video of someone disassembling a toy was shown. Here the instruction simply was to watch the video, without the need to remember the sequence of actions. The rationale of this manipulation is that observational learning was induced in the LEARN condition but not in the CONTROL condition, whereas perceptual input was kept constant. Both conditions compared with a passive baseline (watching a fixation cross) activated the "action recognition network" (including parietal, inferior frontal, and premotor areas but also cerebellum and part of the basal ganglia) [Frey and Gerry (2006), their Fig. 2 (http:// www.jneurosci.org/cgi/content/full/26/ $51 / 13194 / F 2)]$. Interestingly, in the LEARN condition, parts of this network were more strongly activated than in the CONTROL condition [Frey and Gerry (2006), their Fig. 3 (http://www.jneurosci. org/cgi/content/full/26/51/13194/F3)]. In other words, this action recognition system was more strongly activated when participants observed an action with the intention to reproduce it later, compared with when they observed the action without the intention to reproduce it. What is interesting is that also parts of the motor system traditionally regarded as "lower" in the cortical hierarchy such as the dorsal part of premotor cortex or pre-supplementary motor area (SMA) showed this increase in activation (see also Grezes et al., 1999). Interestingly, activation in right intraparietal cortex showed a strong correlation with participants' actual performance on reconstructing the Tinkertoy after watching the video [Frey and Gerry (2006), their Fig. 4 (http://www.jneurosci. org/cgi/content/full/26/51/13194/F4)] .

This latter finding is especially relevant in the context of the second experiment in which the authors specifically looked into the role of sequencing. The same stimuli were used in a different group of participants. Now the task was to either simply watch the videos (CONTROL condition as before) or to watch the videos with the intention to be able to reconstruct the Tinkertoy afterward. The crucial difference with experiment 1 was that no mention was made as to how the toy had to be reconstructed, i.e., about the exact sequence of acts. All that was asked of participants was to recreate the toy. Indeed, behavioral scores indicate that although participants were as good at reconstructing the toy as in experiment 1, they less often used exactly the same sequence of acts as those demonstrated in the video. Similar to the previous experiment, a network of areas in parietofrontal areas (including premotor cortex), the cerebellum and basal ganglia, showed increased activation to the LEARN condition compared 
with the CONTROL condition [Frey and Gerry (2006), their Fig. 5 (http://www. jneurosci.org/cgi/content/full/26/51/ 13194/F5)]. The relationship between offline performance and activity in right intraparietal cortex as reported in experiment 1 was, however, not observed. The authors conclude that this parietal region plays a crucial role in the encoding of a sequence. This is an interesting finding given studies that propose a role for intraparietal cortex in coding the goal of an action (Hamilton and Grafton, 2006). More research is needed, however, to understand how these findings can be related.

That Frey and Gerry found dorsal premotor cortex and especially pre-SMA to be more strongly activated in the "intentional" LEARN condition than in the passive observation condition is in line with a study showing pre-SMA activation specifically correlated to the moment in time at which participants felt the intention to move their finger (Lau et al., 2004). It seems that this premotor structure is involved in the coding of the intention to act [but see Iacoboni et al. (2005) for a different interpretation in a study on the understanding of intentions]. How generally true this is, as well as whether it translates to other domains of cognition, is an important question for the future.

One methodological concern with the present study is that changing task instructions could lead to confounds. It is possible for instance that subjects in the LEARN condition paid much closer attention to the actions than in the CONTROL condition. Along this line, the activation increases might be the result of a more general attentional effect than observational learning. Put differently, the conditions may not have been that perceptually similar after all, if one takes perceptual similarity to also include the "exactness" with which participants observe the stimulus. Although the fact that premotor areas were activated may argue against such an explanation, it is hard to exclude a top-down effect of attention on premotor areas. It could be argued that possible attentional factors were lower in experiment 2. After all, participants "just" had to pay attention to the end-state of the toy, not to every action individually. Unfortunately, activations in the "action recognition areas" closely follow the pattern expected from an attentional explanation: high in experiment 1 , lower in experiment 2 [Frey and Gerry (2006), their Fig. 6 (http://www.jneurosci.org/cgi/content/ full/26/51/13194/F6)]. The similar accuracy scores in the two experiments (89 vs $83 \%$ ) can furthermore be interpreted as evidence for equal nonspecific task demands, albeit indirect evidence. However, it seems only fair to say that different degrees of attention are perhaps inherent to observational learning versus more passive observation.

In conclusion, Frey and Gerry nicely show the involvement of the action recognition system (specifically premotor areas) in coding the intention to learn a sequence of acts. Moreover, their findings extend knowledge about intraparietal cortex in action observation by showing its sensitivity to the sequence of observed motor acts. One part that deserves more attention in future research is the strong social nature of observational learning. In real life, the social relationship we have with an instructor seems important in the success of observational learning. That is, you probably have more faith in your parents teaching you how to handle your shoelaces than in your younger sister who hardly knows how to do it herself.

\section{References}

Frey SH, Gerry VE (2006) Modulation of neural activity during observational learning of actions and their sequential orders. J Neurosci 26:13194-13201.

Grezes J, Costes N, Decety J (1999) . The effects of learning and intention on the neural network involved in the perception of meaningless actions. Brain 122:1875-1887.

Hamilton AF, Grafton ST (2006) Goal representation in human anterior intraparietal sulcus. J Neurosci 26:1133-1137.

Iacoboni M, Molnar-Szakacs I, Gallese V, Buccino G, Mazziotta JC, Rizzolatti G (2005) Grasping the intentions of others with one's own mirror neuron system. PLoS Biol 3:e79.

Lau HC, Rogers RD, Haggard P, Passingham RE (2004) Attention to intention. Science 303:1208-1210. 